
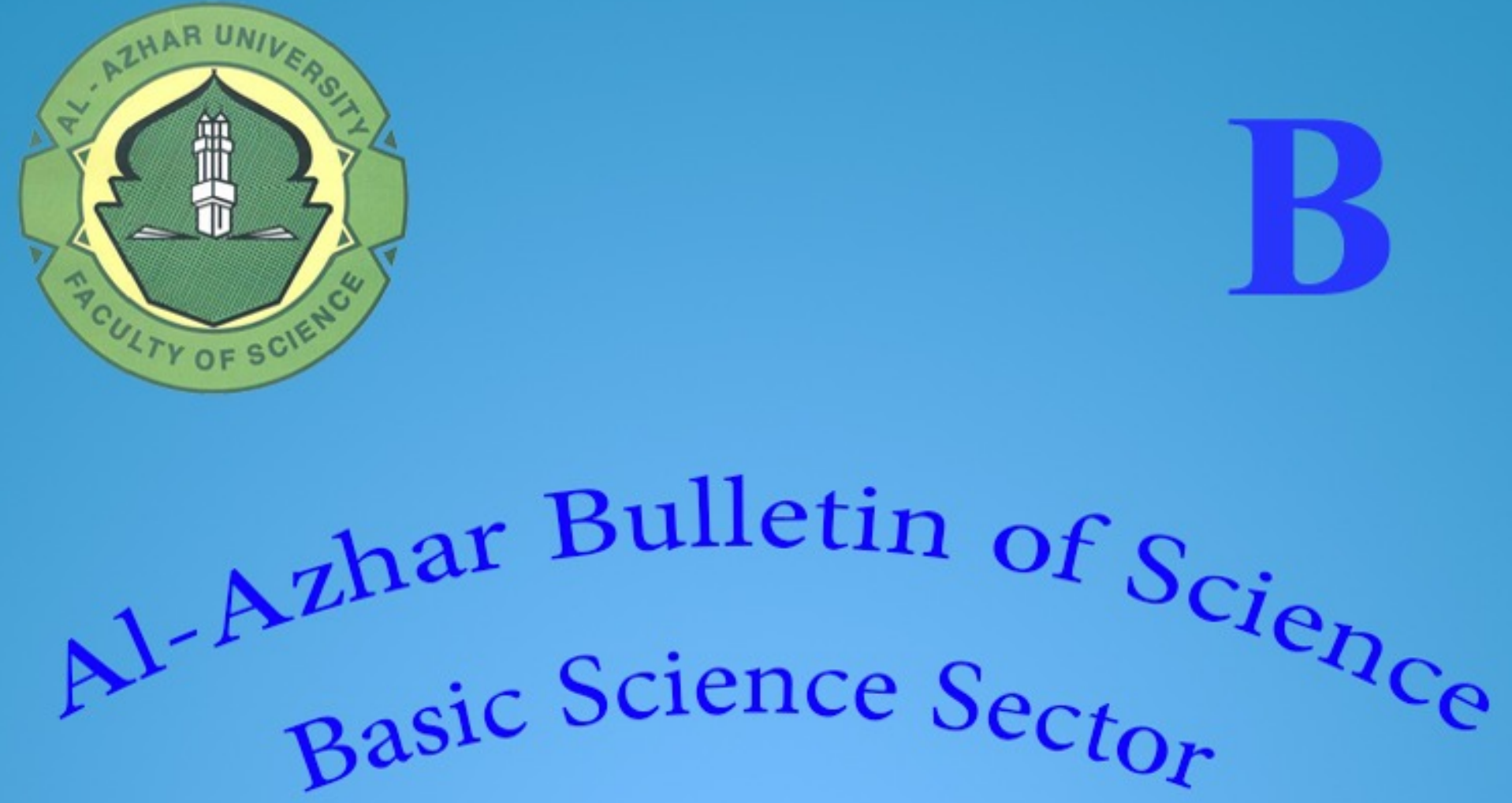

THE OFFICIAL PUBLICATION OF

THE FACULTY OF SCINCE

( MEN , GIRLS \&ASSUIT BRANCH )

AL-AZHAR UNIVERSITY

CAIRO, EGYPT

\title{
ABSB
}
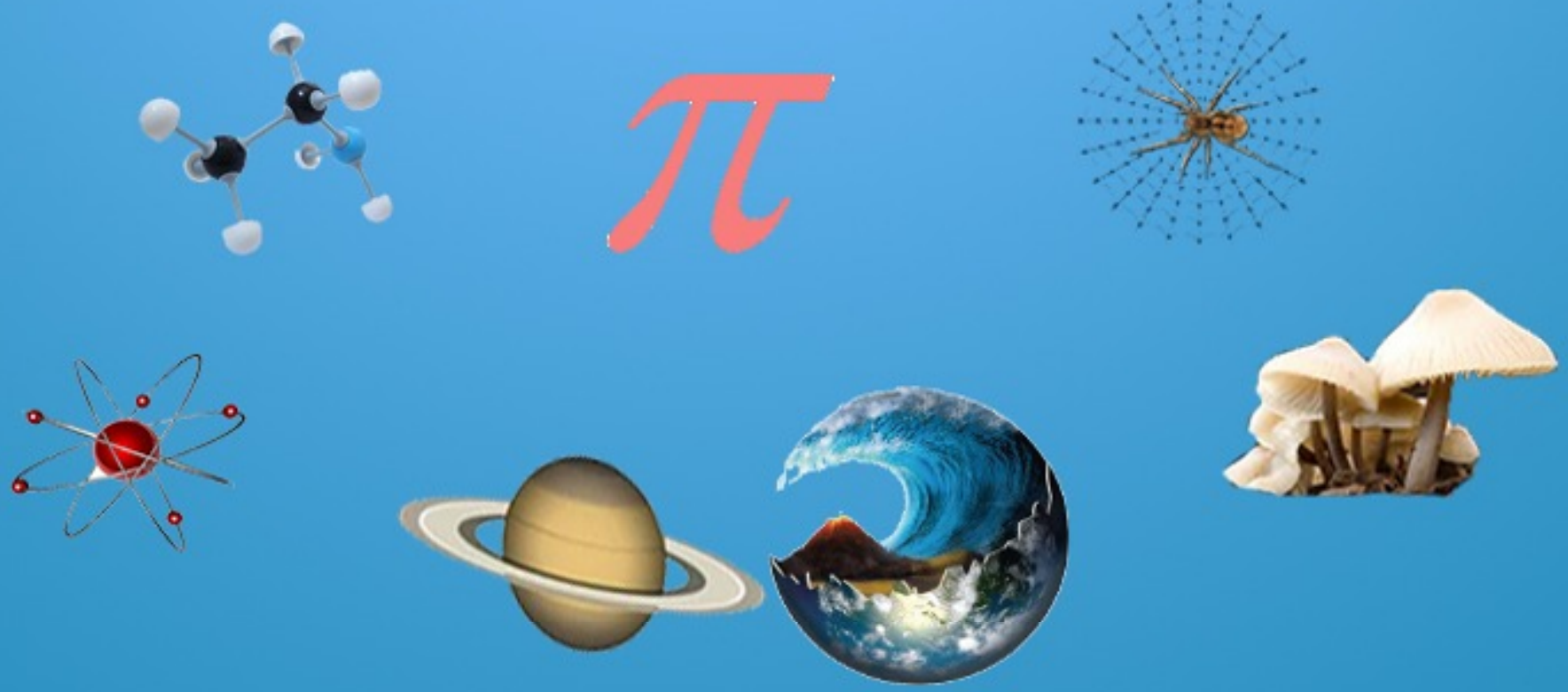


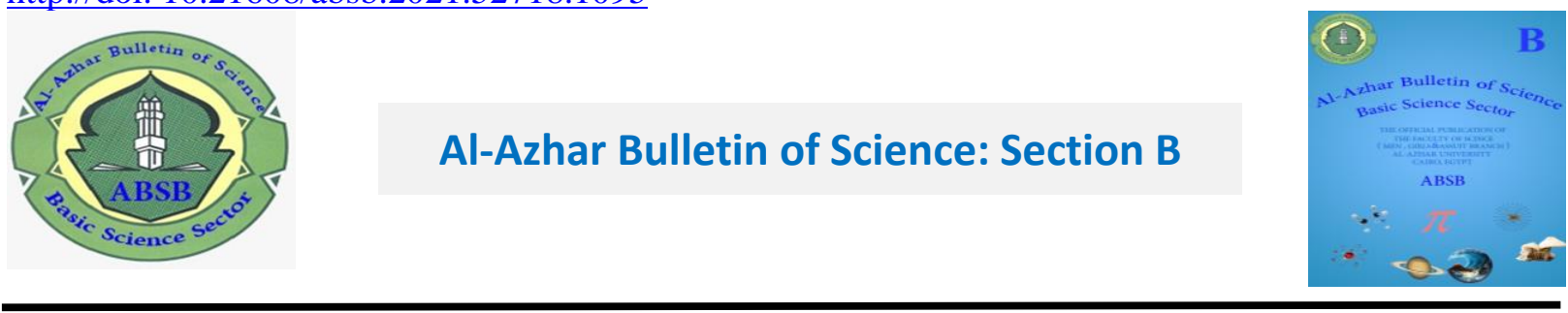

\title{
TOPP-LEONE LOMAX DISTRIBUTION
}

\section{S.S. Radwan*}

Department of Mathematics, Faculty of Science (Girls' Section), Al-Azhar University, Nasr City, Cairo, Egypt.

*Corresponding Author: Salwa_1977@ hotmail.com

Received: 03 Jan 2021; Revised: 01 Feb 2021; Accepted: 09 Feb 2021; Published: 27 Sep 2021

\begin{abstract}
A new compound distribution is extended in this article, the Lomax distribution by using the Topp-Leone generated family. The statistical properties as Quantile function, moments and distribution of order statistics are studied. Maximum likelihood method is discussed and the element of the information matrix, differential entropy are provided. Simulation study is conducted to assess the performance of the parameters of Topp Leone Lomax distribution. An application is used to show that our distribution is better than one based on the Lomax and Exponentiated Lomax distribution.
\end{abstract}

Keywords: Topp-Leone; Lomax distribution; moment generating function; the differential entropy; Information matrix; Simulation study.

\section{Introduction}

Lomax distribution or Pareto typeII distribution, which is heavy tail probability distribution, is useful in business, economics and queuing theory. It is named after K.S.Lomax. Using Topp-Leone distribution [8], Sangsanit and Bodhisuwan[6] introduced a new framework for generating lifetime distributions, called Topp-Leone generated (TLG) family distribution.

Nadarajah and Haghighi [3] discussed a new generalization of the exponential distribution, named Nadarajah- Haghighi distribution and using the Topp-Leone generated family of distribution which was introduced in [6] by Sangsanit \& Bodhisuwan, deduced a new model and discussed all its properties. Also, Yousof and Korkmaz [9] discussed and introduced a new model called the ToppLeone Nadarajah- Haghighi distribution.

A new model called Topp-Leone Lomax distribution has been introduced, where the Lomax distribution is extended by using the Topp-Leone generated family. We discuss in Section (2) the Generalization of new model and some of its properties. In Section (3) we derive some statistical properties as quantile, moments. Maximum likelihood estimation and estimates parameters are derived in (4), Differential entropy is obtained in (5), A simulation study along with real data set are introduced in Sections (6) and (7) respectively to show the flexibility of the new model.

\section{Mixed Topp-Leone Lomax distribution}

A random variable $\mathrm{X}$ is said to have a Lomax distribution if its probability density function (pdf) and cumulative distribution function (cdf) are defined respectively as

$$
\mathrm{g}(\mathrm{x})=\frac{\alpha}{\lambda}\left(1+\frac{\mathrm{x}}{\lambda}\right)^{-(\alpha+1)} \quad \mathrm{x} \geq 0
$$




$$
\mathrm{G}(\mathrm{x})=1-\left(1+\frac{\mathrm{x}}{\lambda}\right)^{-\alpha} \quad \alpha, \lambda>0, \mathrm{x} \geq 0
$$

where ${ }^{\alpha}$ is a shape parameter and $\lambda$ is a scale parameter.

Inserting (1) \& (2) in Topp-Leone generated family distribution which was introduced by Sangsanit, Bodhisuwan[8] given by:

$$
\begin{aligned}
& \mathrm{f}(\mathrm{x})=2 \beta \mathrm{g}(\mathrm{x})[\mathrm{G}(\mathrm{x})]^{\beta-1}[1-G(\mathrm{x})][2-\mathrm{G}(\mathrm{x})]^{\beta-1} \\
& \mathrm{~F}(\mathrm{x})=[\mathrm{G}(\mathrm{x})[2-\mathrm{G}(\mathrm{x})]]^{\beta}
\end{aligned}
$$

where $\beta>0$ is the shape parameter, the pdf \& cdf of TOPP-leone Lomax Distribution (TLL),

$$
\begin{aligned}
& \mathrm{f}(\mathrm{x})=\frac{2 \beta \alpha}{\lambda}\left(1+\frac{\mathrm{x}}{\lambda}\right)^{-(2 \alpha+1)}\left[1-\left(1+\frac{\mathrm{x}}{\lambda}\right)^{-2 \alpha}\right]^{\beta-1} \\
& \mathrm{~F}(\mathrm{x})=\left[1-\left(1+\frac{\mathrm{x}}{\lambda}\right)^{-2 \alpha}\right]^{\beta}
\end{aligned}
$$

where $\alpha, \lambda, \beta>0, x \geq 0$. The model in (5) and (6) will be called Topp-Leone Lomax distribution. The hazard rate function of TLL is given by

$$
\begin{aligned}
& h(\mathrm{x})=\frac{\mathrm{f}(\mathrm{x})}{1-\mathrm{F}(\mathrm{x})} \\
& h(\mathrm{x})=\frac{\frac{2 \beta \alpha}{\lambda}\left(1+\frac{\mathrm{x}}{\lambda}\right)^{-(2 \alpha+1)}\left[1-\left(1+\frac{\mathrm{x}}{\bar{\lambda}}\right)^{-2 \alpha}\right]^{\beta-1}}{1-\left[1-\left(1+\frac{\mathrm{x}}{\bar{\lambda}}\right)^{-2 \alpha}\right]^{\beta}} \quad \alpha, \lambda, \beta>0, x \geq 0
\end{aligned}
$$

Also reliability is defined as

$\mathrm{S}(\mathrm{x})=1-F(\mathrm{x})$,

$\mathrm{S}(\mathrm{x})=1-\left[1-\left(1+\frac{\mathrm{x}}{\lambda}\right)^{-2 \alpha}\right]^{\beta}$

Some graphical representations are introduced, Figs. (1), (2), (3) and (4) show the different shapes of pdf , cdf, Hazard rate function and reliability respectively at different parameter values.

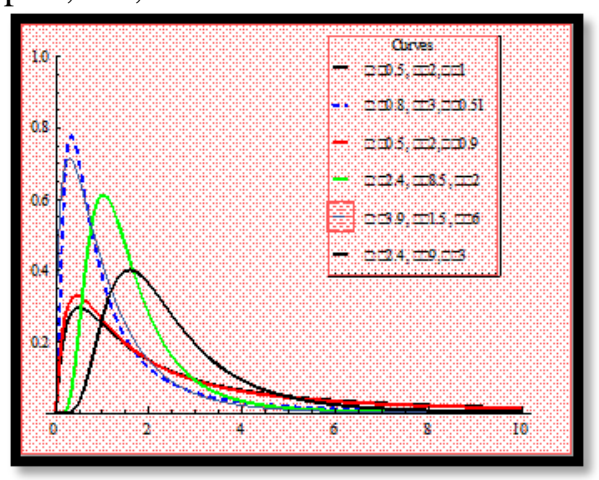

Fig.1. pdf for some parameter values

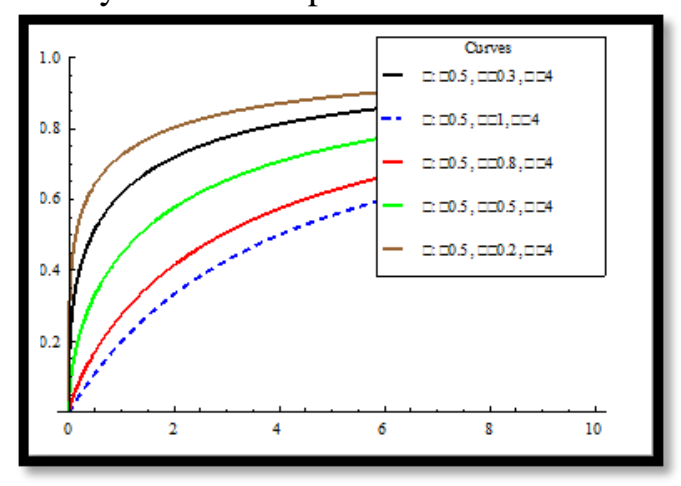

Fig. 2. Cumulative distribution function (cdf) 


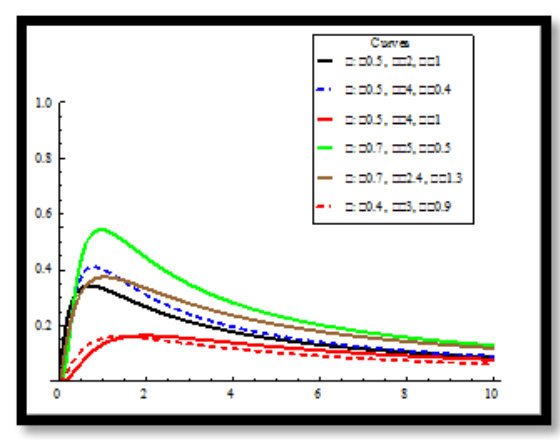

Fig. 3. Hazard rate function

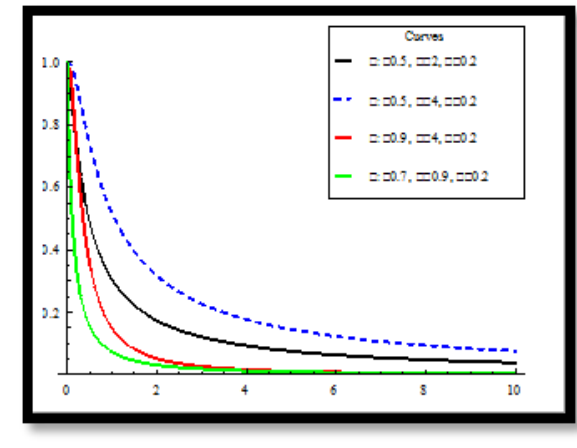

Fig. 4. Reliability function

From the Fig. 3, we observe the hazard rate function increases to the maximum value, decreases steadily.

\section{SOME STATISTICAL PROPERTIES}

\subsection{The Quantile Function}

$\mathrm{u}^{\text {th }}$ quantile function can define by:

$\mathrm{F}[\mathrm{x}]=u, \quad 0<u<1$

From Equation (6)

$$
\begin{gathered}
\mathrm{u}=\left[1-\left(1+\frac{\mathrm{x}}{\lambda}\right)^{-2 \alpha}\right]^{\beta} \quad \mathrm{x} \geq 0, \\
\mathrm{x}_{\mathrm{u}}=\lambda\left(\left[1-\mathrm{u}^{\frac{1}{\beta}}\right]^{\frac{-1}{2 \alpha}}-1\right\}, \quad 0<\mathrm{u}<1
\end{gathered}
$$

For $\mathrm{u}=0.5$, the median is

$$
\mathrm{x}_{0.5}=\lambda\left\{\left[1-(0.5)^{\frac{1}{\beta}}\right]^{\frac{-1}{2 \alpha}}-1\right\}
$$

\subsection{Moments}

If $\mathrm{X}$ is a random variable with pdf \& cdf (5) \& (6), so the $\mathrm{r}^{\text {th }}$ moment is given by

$$
E\left(X^{r}\right)=\int_{x} x^{r} f(x) d x \quad x \geq 0
$$

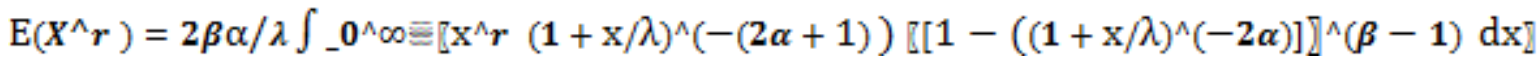
(11)

Applying binomial series

$$
\left[1-\left(1+\frac{\mathrm{x}}{\bar{\lambda}}\right)^{-2 \alpha}\right]^{\beta-1}=\sum_{k=0}^{\beta-1}\left(\begin{array}{c}
\beta-1 \\
k
\end{array}\right)(-1)^{\mathrm{k}}\left[1+\frac{\mathrm{x}}{\lambda}\right]^{-2 \alpha \mathrm{k}} \quad, \beta>1
$$


Then

$$
\mathrm{E}\left(\mathrm{X}^{\mathrm{r}}\right)=\frac{2 \beta \alpha}{\lambda} \sum_{\mathrm{k}=0}^{\beta-1}\left(\begin{array}{c}
\beta-1 \\
\mathrm{k}
\end{array}\right)(-1)^{\mathrm{k}} \frac{\lambda^{\mathrm{r}+1} \Gamma(\mathrm{r}+1) \Gamma(-\mathrm{r}+2(\alpha+\alpha \mathrm{k}))}{\Gamma(1+2 \alpha(\mathrm{k}+1))}
$$

where $-\mathrm{r}+2(\alpha+\alpha \mathrm{k}) \geq 0$,

Put $r=1$, the expected value of TLL distribution is given by

$$
\mathrm{E}(\mathrm{X})=2 \beta \alpha \lambda \sum_{\mathrm{k}=0}^{\beta-1}\left(\begin{array}{c}
\beta-1 \\
\mathrm{k}
\end{array}\right)(-1)^{\mathrm{k}} \frac{1}{[2 \alpha(\mathrm{k}+1)+1][2 \alpha(1+\mathrm{k})]}
$$

by putting $\mathrm{r}=2$ at $(12)$, we get

$$
\left(\begin{array}{c}
\beta-1 \\
\mathrm{k}
\end{array}\right)(-1)^{\mathrm{k}} \frac{1}{([2 \alpha(\mathrm{k}+1)+1][2 \alpha(1+\mathrm{k})][[2 \alpha(1+\mathrm{k})-1])]}
$$

From Equations (13) \& (14) we can obtain the variance of TLL distribution, where

$$
\operatorname{var}(X)=E\left[\mathrm{X}^{2}\right]-(E[\mathrm{X}])^{2}
$$

\subsection{Moment Generating Function}

Moment generating function of TLL distribution from (5) \& (6)

$$
\begin{aligned}
& \mathrm{E}\left(\mathrm{e}^{\mathrm{Xt}}\right)=\int_{\mathrm{x}=0}^{\infty} \mathrm{e}^{\mathrm{Xt}} f(\mathrm{x}) d \mathrm{X} \\
& \mathrm{E}\left(e^{\wedge} \mathrm{Xt}\right)=2 \beta \alpha / \lambda \int 0^{\wedge} \infty \equiv \llbracket\left[e^{\Lambda} \mathrm{Xt}(1+x / \lambda)^{\wedge}(-(2 \alpha+1)) \llbracket\left[1-\left((1+x / \lambda)^{\wedge}(-2 \alpha)\right]\right]^{\wedge}(\beta-1) d x \rrbracket\right. \\
& \mathrm{E}\left(\mathrm{e}^{\mathrm{Xt}}\right)=\frac{2 \beta \alpha}{\lambda} \sum_{k=0}^{\beta-1}\left(\begin{array}{c}
\beta-1 \\
\mathrm{k}
\end{array}\right)(-1)^{k} \int_{0}^{\infty} \mathrm{e}^{\mathrm{xt}}\left(\frac{\lambda+\mathrm{x}}{\lambda}\right)^{-(2 \alpha(1+\mathrm{k})+1)} \mathrm{dx} \\
& \mathrm{E}\left(\mathrm{e}^{\mathrm{Xt}}\right)=2 \beta \alpha \sum_{\mathrm{k}=0}^{\beta-1}\left(\begin{array}{c}
\beta-1 \\
\mathrm{k}
\end{array}\right)(-1)^{k} e^{-\lambda \mathrm{t}}(-\mathrm{t})^{2 \alpha(\mathrm{k}+1)} \lambda^{2 \alpha(\mathrm{k}+1)}[-2 \alpha(\mathrm{k}+1),-t \lambda]
\end{aligned}
$$

\subsection{Distribution of order statistics}

Let $\mathrm{X}_{1: \mathrm{n}} \leq \mathrm{X}_{2 \mathrm{n}} \leq \cdots \leq \mathrm{X}_{\mathrm{n}: \mathrm{n}}$ be the order sample for a continuous population with pdf $\mathrm{f}(\mathrm{x})$. The pdf of $X_{j: n}$ the $j^{\text {th }}$ order statistics is given by

$$
\llbracket f\left(x \rrbracket_{j: n}\right)=\frac{n !}{(j-1) !(n-j) !} f(x)[F(x)]^{j-1}[1-F(x)]^{n-j}
$$

$\llbracket f\left(x \rrbracket_{j: n}\right)=\frac{2 \beta \alpha}{\lambda} \frac{n !}{(j-1) !(n-j) !}\left[1+\frac{x}{\lambda}\right]^{-2(\alpha+1)}\left[1-\left(1+\frac{x}{\lambda}\right)^{-2 \alpha}\right]^{\beta f-1}\left[1-\left[1-\left(1+\frac{x}{\lambda}\right)^{-2 \alpha}\right]^{\beta}\right]^{n-f}$

So, the distribution of the minimum and maximum order statistics of TLL are given by 


$$
\begin{aligned}
& \llbracket f\left(x \rrbracket_{1 \cdot n}\right)=\frac{2 n \alpha \beta}{\lambda}\left[1+\frac{x}{\lambda}\right]^{-(2 \alpha+1)}\left[1-\left(1+\frac{x}{\lambda}\right)^{-2 \alpha}\right]^{\beta-1}\left[1-\left[1-\left(1+\frac{x}{\lambda}\right)^{-2 \alpha}\right]^{\beta}\right]^{n-1} \\
& \llbracket f\left(x \rrbracket_{n-n}\right)=\frac{2 n \alpha \beta}{\lambda}\left[1+\frac{x}{\lambda}\right]^{-2(\alpha+1)}\left[1-\left(1+\frac{x}{\lambda}\right)^{-2 \alpha}\right]^{n \beta-1}
\end{aligned}
$$

\section{Maximum Likelihood estimations}

The parameters of TLL has been estimated by using maximum likelihood estimation. Let $\mathrm{X}_{1}, \mathrm{X}_{2}, \ldots, \mathrm{X}_{\mathrm{n}}$ a random sample has density function given in (5), then likelihood function is given by

$L=\frac{2^{n} \beta^{n} \alpha^{n}}{\lambda^{n}} \prod_{i=1}^{n}\left(1+\frac{x_{i}}{\lambda}\right)^{-(2 \alpha+1)} \prod_{i=1}^{n}\left[1-\left(1+\frac{x_{i}}{\lambda}\right)^{-2 \alpha}\right]^{\beta-1}$

The log likelihood function is given by

$$
\begin{aligned}
& \log L=n \log 2+n \log \beta+n \log \alpha-n \log \lambda-(2 \alpha+1) \sum_{i=1}^{n} \llbracket \log \left(1+\frac{x_{i}}{\lambda} \rrbracket\right)+ \\
& 1+(\beta-1) \sum_{i=1}^{n} \llbracket \log \left(1-\left(1+\frac{x_{i}}{\lambda}\right)^{-2 \alpha} \rrbracket\right)
\end{aligned}
$$

Differentiating the Equation (18) with respect to $\beta, \alpha, \lambda$ we obtain score vector $\left(\mathbb{Q} U_{-} \beta, U_{-} \alpha_{,} U_{-} \lambda\right) \rrbracket^{\wedge} T$, where elements of score vector are given by

$$
\begin{gathered}
\mathrm{U}_{\beta}=\frac{\partial \operatorname{LogL}}{\partial \beta}=\frac{\mathrm{n}}{\beta}+\sum_{i=1}^{\mathrm{n}} \log \left(1-\left(1+\frac{\mathrm{x}_{\mathrm{i}}}{\lambda}\right)^{-2 \alpha}\right) \\
\mathrm{U}_{\alpha}=\frac{\partial \operatorname{LogL}}{\partial \alpha}=\frac{\mathrm{n}}{\alpha}-2 \sum_{\mathrm{i}=1}^{\mathrm{n}} \log \left(1+\frac{\mathrm{x}_{\mathrm{i}}}{\lambda}\right)+(\beta-1) \sum_{\mathrm{i}=1}^{\mathrm{n}} \frac{2\left[\log \left(1+\frac{\mathrm{x}_{\mathrm{i}}}{\lambda}\right)\right]\left(1+\frac{\mathrm{x}_{\mathrm{i}}}{\lambda}\right)^{-2 \alpha}}{\left(1-\left(1+\frac{\mathrm{x}_{\mathrm{i}}}{\lambda}\right)^{-2 \alpha}\right)} \\
\mathrm{U}_{\lambda}=\frac{\partial \mathrm{LogL}}{\partial \lambda}=\frac{-\mathrm{n}}{\lambda}+(2 \alpha+1) \sum_{\mathrm{i}=1}^{\mathrm{n}} \frac{\mathrm{x}_{\mathrm{i}} \lambda^{2}\left(1+\frac{\mathrm{x}_{\mathrm{i}}}{\lambda}\right)}{(\beta-1)} \sum_{\mathrm{i}=1}^{\mathrm{n}} \frac{2 \alpha \mathrm{x}_{\mathrm{i}}\left(1+\frac{\mathrm{x}_{\mathrm{i}}}{\lambda}\right)^{-(2 \alpha+1)}}{\left.\left(1+\frac{\mathrm{x}_{\mathrm{i}}}{\lambda}\right)^{-2 \alpha}\right)}
\end{gathered}
$$

To obtain the MLE of parameters by setting non-linear system of equations $\mathrm{U}_{\beta}, \mathrm{U}_{\alpha}, \mathrm{U}_{\lambda}=\mathbf{0}$,solving them and choosing the solution which give the best value of the maximum likelihood equation. Then $3 \times 3$ Fisher information matrix is derived by differentiating Equation (19), (20) and (21) once more with respect to the three parameters $\beta, \alpha$ and $\lambda$ to give 
22

S.S. Radwan

$$
\varphi=\left(\begin{array}{ccc}
\mathrm{U}_{\beta \beta} & \mathrm{U}_{\beta \alpha} & \mathrm{U}_{\beta \lambda} \\
\mathrm{U}_{\alpha \beta} & \mathrm{U}_{\alpha \alpha} & \mathrm{U}_{\alpha \lambda} \\
\mathrm{U}_{\lambda \beta} & \mathrm{U}_{\lambda \alpha} & \mathrm{U}_{\lambda \lambda}
\end{array}\right)
$$

The elements of this matrix are as follows

$$
\begin{aligned}
& \mathrm{U}_{\beta \beta}=-\frac{n}{\beta^{2}} \\
& \mathrm{U}_{\beta \alpha}=\sum_{\mathrm{i}=1}^{n} \frac{2\left(\log \left(1+\frac{\mathrm{x}_{\mathrm{i}}}{\lambda}\right)\right)\left(1+\frac{\mathrm{x}_{\mathrm{i}}}{\lambda}\right)^{-2 \alpha}}{\left[1-\left(1+\frac{\mathrm{x}_{\mathrm{i}}}{\lambda}\right)^{-2 \alpha}\right]} \\
& \mathrm{U}_{\beta \lambda}=\sum_{\mathrm{i}=1}^{\mathrm{n}}-\frac{2 \alpha \mathrm{x}_{\mathrm{i}}\left(1+\frac{\mathrm{x}_{\mathrm{i}}}{\lambda}\right)^{-2 \alpha-1}}{\lambda^{2}\left[1-\left(1+\frac{\mathrm{x}_{\mathrm{i}}}{\lambda}\right)^{-2 \alpha}\right]} \\
& \mathrm{U}_{\alpha \beta}=\sum_{\mathrm{i}=1}^{n} \frac{\left(\log \left(1+\frac{\mathrm{x}_{\mathrm{i}}}{\lambda}\right)\right)\left(1+\frac{\mathrm{x}_{\mathrm{i}}}{\lambda}\right)^{-2 \alpha}}{\left[1-\left(1+\frac{\mathrm{x}_{\mathrm{i}}}{\lambda}\right)^{-2 \alpha}\right]}
\end{aligned}
$$

$$
\mathrm{U}_{\alpha \alpha}=-\frac{\mathrm{n}}{\alpha^{2}}+(\beta-1) \sum_{\mathrm{i}=1}^{\mathrm{n}} \mathbb{[}\left[\frac{-4 \log \left(1+\frac{\mathrm{x}_{\mathrm{i}}}{\lambda}\right)^{2}\left(1+\frac{\mathrm{x}_{\mathrm{i}}}{\lambda}\right)^{-2 \alpha}}{\left[1-\left(1+\frac{\mathrm{x}_{\mathrm{i}}}{\lambda}\right)^{-2 \alpha}\right]}\left\{\frac{\left(1+\frac{\mathrm{x}_{\mathrm{i}}}{\lambda}\right)^{-2 \alpha}}{\left[1-\left(1+\frac{\mathrm{x}_{\mathrm{i}}}{\lambda}\right)^{-2 \alpha}\right]}-1\right\}\right]
$$

$$
=-2 \sum_{i=1}^{n} \frac{-x_{i}}{\lambda^{2}\left(1+\frac{x_{i}}{\lambda}\right)}+(\beta-1) \sum_{i=1}^{n}\left[\frac{4 \alpha x_{i} \log \left(1+\frac{x_{i}}{\lambda}\right)\left(1+\frac{x_{i}}{\lambda}\right)^{-2 \alpha-1}}{\lambda^{2}\left[1-\left(1+\frac{x_{i}}{2}\right)^{-2 \alpha}\right]} \rrbracket\left\{\frac{\left(1+\frac{x_{i}}{\lambda}\right)^{-2 \alpha}}{\left.\mid 1-\left(1+\frac{x_{i}}{2}\right)^{-2 \alpha}\right\rceil}+1\right\}-\frac{2 x_{i}\left(1+\frac{x_{i}}{\lambda}\right)^{-2 \alpha-1}}{\lambda^{2}\left[1-\left(1+\frac{x_{i}}{2}\right)^{-2 \alpha]}\right.} \mid\right.
$$

$$
\begin{aligned}
& \mathrm{U}_{\lambda \beta}=\sum_{i=1}^{n} \frac{-2 \alpha x_{i}\left(1+\frac{x_{i}}{\lambda}\right)^{-2 \alpha-1}}{\lambda^{2}\left[1-\left(1+\frac{x_{i}}{\lambda}\right)^{-2 \alpha}\right]} \\
& \mathrm{U}_{\lambda \alpha}=-2 \sum_{i=1}^{n} \frac{-x_{i}}{\lambda^{2}\left(1+\frac{x_{i}}{\lambda}\right)}+(\beta-1) \sum_{i=1}^{n}\left[\frac{4 \alpha x_{i} \log \left(1+\frac{x_{i}}{\lambda}\right)\left(1+\frac{x_{i}}{\lambda}\right)^{-2 \alpha-1}}{\lambda^{2}\left[1-\left(1+\frac{x_{i}}{\lambda}\right)^{-2 \alpha}\right]}\left\{\frac{\left(1+\frac{x_{i}}{\lambda}\right)^{-2 \alpha}}{\left[1-\left(1+\frac{x_{i}}{\lambda}\right)^{-2 \alpha}\right]}+1\right\}-\frac{2 x_{i}\left(1+\frac{x_{i}}{\lambda}\right)^{-2 \alpha-1}}{\lambda^{2}\left[1-\left(1+\frac{x_{i}}{\lambda}\right)^{-2 \alpha}\right]}\right]
\end{aligned}
$$




$$
\left[\frac{\mathrm{x}_{\mathrm{i}}}{\lambda^{3}\left(1+\frac{\mathrm{x}_{\mathrm{i}}}{\lambda}\right)}\right]\left\{\frac{-\mathrm{x}_{\mathrm{i}}}{\lambda\left(1+\frac{\mathrm{x}_{\mathrm{i}}}{\lambda}\right)}+2\right\}(\beta-1) \sum_{\mathrm{i}=1}^{n}\left[\frac{2 \alpha \mathrm{x}_{\mathrm{i}}\left(1+\frac{\mathrm{x}_{\mathrm{i}}}{\lambda}\right)^{-2 \alpha-1}}{\lambda^{3}\left[1-\left(1+\frac{\mathrm{x}_{\mathrm{i}}}{\lambda}\right)^{-2 \alpha}\right]\left\{\frac{-2 \alpha \mathrm{x}_{i}\left(1+\frac{\mathrm{x}_{\mathrm{i}}}{\lambda}\right)^{-2 \alpha-1}}{\lambda\left[1-\left(1+\frac{\mathrm{x}_{\mathrm{i}}}{\lambda}\right)^{-2 \alpha}\right]}+\frac{(-2 \alpha-1) x_{\bar{i}}}{\lambda\left(1+\frac{x_{i}}{\lambda}\right)}+2\right\}}\right]
$$

\section{The differential entropy}

The differential entropy is a concept in information theory that began as an attempt by Shannon to extend the idea of entropy. Let $\mathrm{X}$ be random variable from the TLL distribution, the differential entropy $\mathrm{A}(\mathrm{x})$ can be defined as:

$$
A(x)=-\int_{x} f(x) \log f(x) d x
$$

From Equation (5) the differential entropy of (TLL) model can be written as

$$
A(\mathrm{x})=-\int 0^{n} \cos =\left[2 \beta \alpha / \lambda(1+x / \lambda)^{n}(-(2 \alpha+1))\right]\left[1-\left((1+x / \lambda)^{n}(-2 \alpha)\right]\right]^{\wedge}(\beta-1) \quad * \log \left[2 \beta \alpha / \lambda(1+\mathrm{x} / \lambda)^{n}(-(2 \alpha+1))\right][1-((1
$$

$$
\begin{aligned}
& A(x)=-\int 0_{-}^{\wedge} \infty \equiv\left[2 \beta \alpha / \lambda(1+x / \lambda)^{\wedge}(-(2 \alpha+1))\left[\left[1-\left((1+x / \lambda)^{\wedge}(-2 \alpha)\right]\right]^{\wedge}(\beta-1) \quad *\{\log 2+\log \beta+\log \alpha-\log \lambda-(2 \alpha\right.\right. \\
& A(x) \\
& =-\left[\log 2+\log \beta+\log \alpha-\log \lambda-2 \alpha \beta(2 \alpha+1) \sum^{\beta-1} \sum^{\infty} \frac{(-1)^{\mathrm{k}+\mathrm{L}-1}}{\mathrm{~L}}\left(\begin{array}{c}
\beta-1 \\
\mathrm{k}
\end{array}\right) \frac{\Gamma(\mathrm{L}+1)}{2 \alpha(\mathrm{k}+1)(2 \alpha(\mathrm{k}+1)+1)}+2 \alpha \beta(\beta-1)\right.
\end{aligned}
$$

\section{Simulation study}

Monte Carlo simulation study is conducted to see the performance of MLE of TLL . All results were obtained from 1000 Monte Carlo replication simulation. The random number generation is obtained from quantile function, given by (10). By generating $\mathrm{N}=1000$ samples sizes $10,25,50 \& 75$ of TLL .The mean, variance, bias and mean squared error(MSE) for each parameter was computed using matrix likelihood estimation. It is observed that the mean, variance and MSEs of the parameters $\beta, \alpha, \lambda$ decreases as the sample size increases. All results of this simulation are recorded in Table1.

Table1: The empirical Means, Variances, Biases and MSEs of TLL distribution.

\begin{tabular}{|c|l|c|c|c|c|}
\hline$N$ & Parameter & Mean & Variance & Bias & MSE \\
\hline 10 & $\beta$ & 4.9089 & 2.6016 & -0.2511 & 2.6647 \\
& $\alpha$ & 1.5069 & 1.0200 & 1.2699 & 2.6328 \\
& $\lambda$ & 0.50 .52 & 0.2492 & 0.4827 & 0.4822 \\
\hline 25 & $\beta$ & 4.7373 & 1.7896 & -0.0226 & 1.7901 \\
& $\alpha$ & 0.9687 & 0.6011 & 0.7317 & 1.1366 \\
& $\lambda$ & 0.2485 & 0.2285 & 0.2035 & 0.2699 \\
\hline 50 & $\beta$ & 4.6469 & 1.3256 & -0.3130 & 1.4236 \\
& $\alpha$ & 0.7269 & 0.1424 & 0.48999 & 0.3825 \\
& $\lambda$ & 0.1269 & 0.0629 & 0.08198 & 0.06967 \\
\hline
\end{tabular}

\section{Real data application}

Some mathematical examples were introduced; the maximum likelihood method is applied to estimate the parameters of our model and following criteria were used: Negative Log-Likelihood 
value, Akaike Information Criteria (AIC), Bayesian Information Criteria (BIC), Corrected Akaike Information Criteria (AICC), Hannan \& Quinn Information Criteria (HQIC). A comparison was made using Topp- Leone Lomax , Lomax and Exponentiated Lomax distribution[1].

where

$$
\begin{aligned}
& \mathrm{AIC}=2 \boldsymbol{k}-2 \boldsymbol{l}, \\
& \mathrm{BIC}=-2 l+\mathrm{kLog} n_{s} \\
& \mathrm{AICC}={ }^{\mathrm{AIC}}+\frac{2 \mathrm{k}(\mathrm{k}+1)}{\mathrm{n}-\mathrm{k}-1}, \\
& \mathrm{HIQC}=2 \mathrm{kLog}(\log (\mathrm{n}))-2 l,
\end{aligned}
$$

Where $\mathrm{k}$ is the number of parameters and $\mathrm{n}$ is the number of observations.

The data of the example about strengths of $1.5 \mathrm{~cm}$ glass fibers at the UK National Physical Laboratory. Data given in Table 2 has previously been used by Smith and Naylor [6]. The observations are as follows:

\begin{tabular}{|c|c|c|c|c|c|c|}
\hline Distribution & Max. Likelihood Estimates & -1 & AIC & BIC & AICC & HQIC \\
\hline Lomax & $\hat{\alpha}=0.9145, \hat{\lambda}=0.746282$ & 119.07 & 242.14 & 246.426 & 242.34 & 243.822 \\
\hline $\begin{array}{l}\text { Exponentiated } \\
\text { Lomax }\end{array}$ & $\hat{\alpha}=0.02577, \hat{\lambda}=1.029 * \mathbf{1 0}^{6}{ }^{6} \widehat{\boldsymbol{\beta}}=0.0554$ & 266.03 & 538.06 & 544.48 & 538.46 & 540.58 \\
\hline $\begin{array}{l}\text { Topp- Leone } \\
\text { Lomax }\end{array}$ & $\hat{\alpha}=5.428, \hat{\lambda}=2.9922, \hat{\beta}=54.280$ & 35.2935 & 76.58 & 83.015 & 76.98 & 79.114 \\
\hline
\end{tabular}

Table 2:

\begin{tabular}{llllllllll}
\hline $\mathbf{0 . 5 5}$ & $\mathbf{0 . 7 4}$ & $\mathbf{0 . 7 7}$ & $\mathbf{0 . 8 1}$ & $\mathbf{0 . 8 4}$ & $\mathbf{1 . 2 4}$ & $\mathbf{0 . 9 3}$ & $\mathbf{1 . 0 4}$ & $\mathbf{1 . 1 1}$ & $\mathbf{1 . 1 3}$ \\
\hline $\mathbf{1 . 3 0}$ & 1.25 & 1.27 & 1.28 & 1.29 & 1.48 & 1.36 & 1.39 & 1.42 \\
$\mathbf{1 . 5 1}$ & 1.49 & 1.49 & 1.50 & 1.50 & 1.55 & 1.52 & 1.53 & 1.54 \\
$\mathbf{1 . 6 1}$ & 1.58 & 1.59 & 1.60 & 1.61 & 1.63 & 1.61 & 1.61 & 1.62 & 1.55 \\
$\mathbf{1 . 6 7}$ & 1.64 & 1.66 & 1.66 & 1.66 & 1.70 & 1.68 & 1.68 & 1.69 & 1.70 \\
$\mathbf{1 . 7 8}$ & 1.73 & 1.76 & 1.76 & 1.77 & 1.89 & 1.81 & 1.82 & 1.84 & 1.84 \\
$\mathbf{2 . 0 0}$ & 2.01 & 2.24 & & & & & & & \\
\hline
\end{tabular}

Table 3: The criteria for the data set.

By using a real data set in another example shows the TLL is better model than one based on Lomax and Exponentiated Lomax distribution. The data set given in Table4 taken from Lawless [2]. The data are number of million revolutions before failure for each of the 23 ball bearings in the life tests

\begin{tabular}{|c|c|c|c|c|c|c|}
\hline Distribution & Max. Likelihood Estimates & -1 & AIC & BIC & $\overline{\mathrm{AICC}}$ & HQIC \\
\hline Lomax & $\hat{\alpha}=0.1573, \hat{\lambda}=0.1104$ & 161.047 & 326.094 & 328.364 & 326.694 & 326.665 \\
\hline $\begin{array}{l}\text { Exponentiated } \\
\text { Lomax }\end{array}$ & $\hat{\alpha}=0.03398, \widehat{\lambda}=1 . \mathbf{3 1} * \mathbf{1 0}^{6}{ }^{6} \widehat{\boldsymbol{\beta}}=0.075$ & 178.013 & 362.026 & 365.432 & 363.289 & 362.872 \\
\hline $\begin{array}{l}\text { Topp- Leone } \\
\text { Lomax }\end{array}$ & $\hat{\alpha}=1.34 \hat{\lambda}=19 \times 242_{x} \hat{\beta}=30.77$ & 114.423 & 234.846 & 238.252 & 236.109 & 235.702 \\
\hline
\end{tabular}

Table 4.

\begin{tabular}{llllll}
\hline $\mathbf{1 7 . 8 8}$ & $\mathbf{2 8 . 9 2}$ & $\mathbf{3 3 . 0 0}$ & $\mathbf{4 1 . 5 2}$ & $\mathbf{4 2 . 1 2}$ & $\mathbf{4 5 . 6 0}$ \\
\hline $\mathbf{4 8 . 8 0}$ & 51.84 & 51.96 & 54.12 & 55.56 & 67.80 \\
$\mathbf{6 8 . 4 4}$ & 68.64 & 68.88 & 84.12 & 93.12 & 98.64 \\
$\mathbf{1 0 5 . 1 2}$ & 105.84 & 127.92 & 128.04 & 173.40 & \\
\hline
\end{tabular}

Table 5: The criteria for the data set 
From Tables 3 and 5, the new model Topp-Leone Lomax distribution fits better than the other distributions, where it gives the minimum value for Log likelihood ,AIC, BIC, AICC \& HQIC criterions.

\section{Conclusions}

Topp-Leone Lomax distribution which extends the Lomax distribution by using the Topp-Leone generated family was introduced. Several properties were derived, quantile function, moments, distribution of order statistics and the differential entropy. Parameters are estimated by maximum likelihood method. Real data sets are applied to demonstrate that the TLL has the lowest AIC, AICC, BIC and HQIC statistics among all other models.

\section{References}

[1] Hamdy MS, The Exponentiated Lomax Distribution: Different Estimation Methods, American journal of Applied Mathmatics and Statistics,2014;2(6):364-8. http://pubs.sciepub.com/ajams/2/6/2 doi:10.12691/ajams2-6-2

[2] Lawless JF, Statistical Models and Methods for Lifetime Data, John Wiley and Sons, NewYork,1982 https://onlinelibrary.wiley.com/doi/pdf/10.1002/9781118033005

[3] Nadarajah S, and Haghighi F, An Extension of the Exponential distribution Statistics, 2011;45:543-58. https://www.tandfonline.com/doi/full/10.1080/02331881003678678

[4] Oguntunde PE, Ahmed MT, Adejumo AO and Odetunmibi OA, A New Generalization of the Lomax Distribution with Increasing, Decreasing and Constant Failure Rate, Modelling and Simulation in Engineering, Article ID6043169. 2017, https://downloads.hindawi.com/journals/mse/2017/6043169

[5] Rezaei S, Sadr BB, Alizadeh M and Nadarajah S, Topp_Leone generated family of distributions: properties and applications, Communications in Statistics-Theory and Method, 2015; 46(6): 2893-909. https://www.tandfonline.com/doi/abs/10.1080/03610926.2015.1053935

[6] Sangsanit $\mathrm{Y}$ and Bodhisuwan W, The Topp-Leone generator of distributions: Properties and inferences", Songklanakarin J Sci Technol, 2016;38:537-48.

https://doaj.org/article/25b6d70e6bf0442880c392d3a8742a42

[7] Smith RL, and Naylor JC, A comparison of maximum likelihood and Bayesian estimators for the threeparameter Weibull distribution, Applied Statistics, ,1987; 36: 258-69. https://rss.onlinelibrary.wiley.com/doi/abs/10.2307/2347795

[8] Topp CW, and Leone FC, A family of J-shaped frequency functions. Journal of American Statistical Association, 1955;50: 209-19.

[9] Yousof HM and Korkmaz MC, Topp-Leone Nadarajah-Haghighi distribution, Journal of Statisticians. Statistics and Actuarial Science, IDIA10, 2017; 2: 119-28. https://www.researchgate.net/publication/322385797_Topp-Leone

$$
\begin{aligned}
& \text { توزيع توبليون لوماكس } \\
& \text { د.سلوى سعيد رضو ان محمود } \\
& \text { كليه العلوم بنات جامعه الازهر (فرع القاهره) }
\end{aligned}
$$

Salwa_1977@hotmail.com

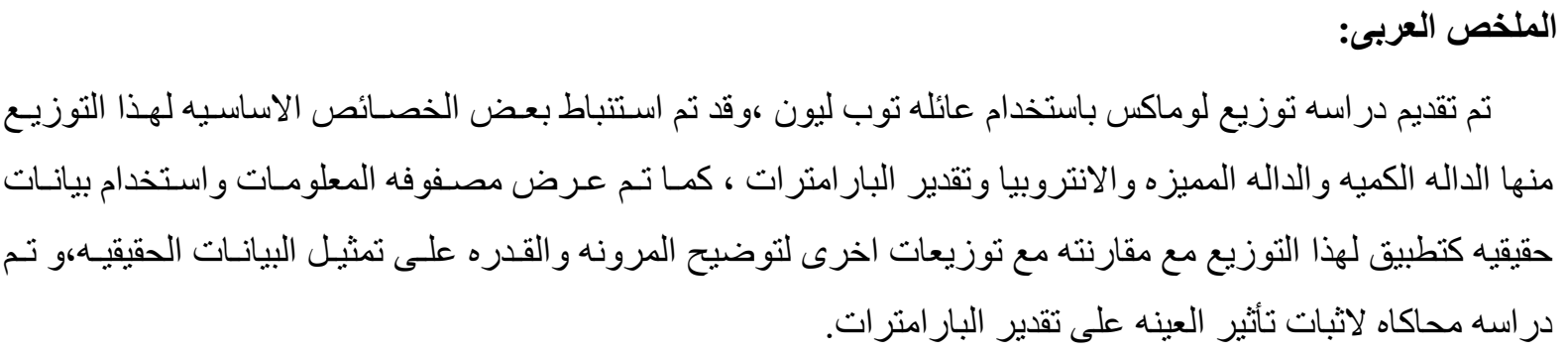

